Check for updates

Cite this: New J. Chem., 2019, 43, 10750

Received 30th April 2019 Accepted 12th June 2019 DOI: 10.1039/c9nj02229e

rsc.li/njc

\section{Acylseleno- and acylthioureato complexes of gold(I) N-heterocyclic carbenes $\dagger$}

\author{
Julia Kuchar, ${ }^{a}$ Jörg Rust, ${ }^{b}$ Christian W. Lehmann ${ }^{b}$ and Fabian Mohr (D)*a \\ A series of gold(I) $\mathrm{N}$-heterocyclic carbene complexes containing monoanionic acylchalcogenourea \\ (chalcogen $=\mathrm{S}, \mathrm{Se}$ ) ligands was prepared and characterised by various spectroscopic methods and X-ray \\ diffraction. The compounds were conveniently accessible in good yields through a one-pot reaction \\ involving $[\mathrm{AuCl}(\mathrm{tht})]$ (tht = tetrahydrothiophene), the imidazolium salt and the acylchalcogenourea in the \\ presence of excess base. The X-ray diffraction experiments showed that in these complexes, the normally \\ chelating ligands adopt the rare $\mathrm{kS} / \mathrm{Se}$-coordination mode in which only the chalcogen is bound to gold.
}

\section{Introduction}

Acylselenoureas are bidentate Se,O-ligands, which, upon deprotonation typically form six-membered chelate rings with a large variety of metals. Examples for such compounds include bis(chelates) with the divalent transition metal ions $\mathrm{Cu}^{2+}, \mathrm{Ni}^{2+}, \mathrm{Pd}^{2+}, \mathrm{Pt}^{2+}, \mathrm{Zn}^{2+}$ and $\mathrm{Cd}^{2+} \cdot{ }^{1-10}$ There are also examples of tris(chelates) with the trivalent metal ions $\mathrm{Fe}^{3+}, \mathrm{Co}^{3+}$ and $\mathrm{In}^{3+} \cdot{ }^{1-13}$ Amongst the main group elements, bis(chelates) containing lead(II) have been of recent interest as single-source precursors for various PbSe nanomaterials. ${ }^{14,15}$ However, examples of such species containing silver and gold are still quite rare. We have previously reported the first $\mathrm{Ag}^{+}$and $\mathrm{Au}^{+}$ compounds containing acylselenoureato and acylthioureato ligands together with a detailed study of their biological activity. ${ }^{16-19}$ We also demonstrated that square planar gold(III) complexes with acylselenoureato ligands are accessible when a dicarbanionic co-ligand is present. ${ }^{20}$ This stabilizing co-ligand is essential to prevent reduction of gold by the acylselenourea during the reaction. Given our interest in gold complexes with selenium and sulfur ligands, ${ }^{21}$ we communicate here the synthesis of some gold(I) complexes containing acylselenoureas and acylthioureas with an N-heterocyclic carbene co-ligand by a simple one-pot process.

\section{Results and discussion}

Initially, we examined three different routes towards the desired NHC-gold(I) acylchalcogenoureato complexes: (1) the reaction of

\footnotetext{
${ }^{a}$ Fakultät für Mathematik und Naturwissenschaften, Anorganische Chemie, Bergische Universität Wuppertal, Gaußstr. 20, 42119 Wuppertal, Germany. E-mail: fmohr@uni-wuppertal.de; Web: www.aoc.uni-wuppertal.de/mohr

${ }^{b}$ Chemische Kristallographie und Elektronenmikroskopie, Max-Planck-Institut für Kohlenforschung, Kaiser-Wilhelm-Platz 1, 45470 Mülheim an der Ruhr, Germany $\dagger$ CCDC 1907692, 1907635 and 1907634. For crystallographic data in CIF or other electronic format see DOI: 10.1039/c9nj02229e
}

the acylchalcogenoureas with [AuCl(IPr)] $[\mathrm{IPr}=1,3-\operatorname{di}(2,6-$ diisopropylphenyl)imidazolylidene] in the presence of base, (2) the reaction of the acylchalcogenoureas with [AuOH(IPr)] and (3) by transmetallation of [AuCl(IPr)] with the corresponding silver(I) acylchalcogenoureato complexes. ${ }^{17}$ To our surprise, the latter two routes afforded either no products at all or we observed formation of dark, inseparable mixtures. In particular, the lack of reactivity of the gold(I) hydroxy complex $[\mathrm{AuOH}(\mathrm{IPr})]$ in this system is somewhat unexpected. The Nolan group has previously demonstrated that this reagent readily reacts with a variety of species containing acidic protons to form the corresponding gold(I) complexes. ${ }^{22}$ Given that the reaction between the acylchalcogenoureas and [AuCl(IPr)] showed the most promising results, we planned to further simplify and generalise the synthesis route by preparing [AuCl(IPr)] in situ directly from the imidazolium salt and [AuCl(tht)] (tht = tetrahydrothiophene) in the presence of base and the acylchalcogenoureas. Gratifyingly, the one-pot reaction of $[\mathrm{AuCl}(\mathrm{tht})]$ with the acylchalcogenoureas and the imidazolium salt in the presence of two equivalents of $\mathrm{KO}^{t} \mathrm{Bu}$ afforded the desired complexes in two hours at room temperature in pure form and good yields (Scheme 1).

Complexes 1-4 were isolated as air-, light- and moisturestable colourless or yellow compounds in yields greater than $60 \%$. The proton NMR spectra of the compounds lack the signal of the NH-proton, consistent with the presence of the deprotonated acylchalcogenourea ligands. Furthermore, the signal due to the carbenic carbon-atom bound to gold can be observed at around $186 \mathrm{ppm}$ (in selenium compounds 1 and 2) or 183 ppm (in sulfur compounds 3 and 4 ) in their ${ }^{13} \mathrm{C}$ NMR spectra. These values are higher than that found in the starting material [AuCl(IPr)] (175 ppm), ${ }^{23}$ confirming that substitution of the chlorido ligand has indeed occurred. Similar chemical shifts for the carbene-carbon-atom have been observed in other 


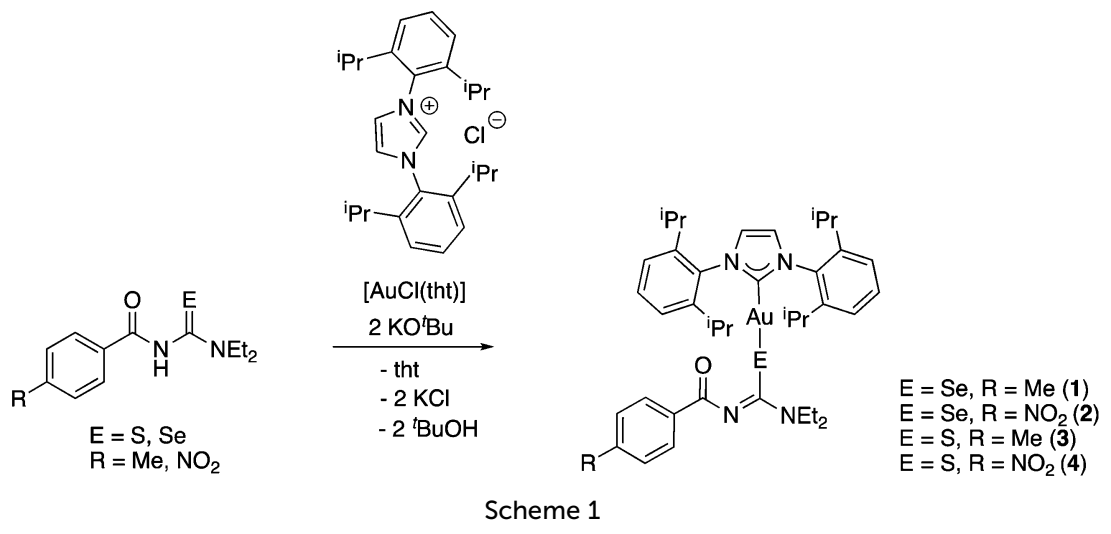

gold(I) NHC-complexes containing anionic chalcogen ligands. ${ }^{24,25}$ For the selenium compounds we were able to record ${ }^{77} \mathrm{Se}$ NMR spectra, which showed singlet resonances shifted upfield by $371 \mathrm{ppm}(\mathbf{1})$ and $380 \mathrm{ppm}$ (2), relative to those of the acylselenoureas themselves. ${ }^{18}$ For comparison, the singlet resonances of the triphenylphosphine counterparts of $\mathbf{1}$ and $\mathbf{2}$ are shifted upfield by $279 \mathrm{ppm}$ and $266 \mathrm{ppm}$, respectively. ${ }^{16}$ This nicely illustrates the electronic differences that phosphine $\left(\mathrm{Ph}_{3} \mathrm{P}\right)$ or carbene (IPr) ligands trans to the selenium atom have on its ${ }^{77}$ Se chemical shift.

We managed to grow crystals of complexes 1,2 and 4 suitable for an X-ray diffraction experiment. The compounds crystallise in the monoclinic crystal system in the space groups $P 2_{1} / n, P 2_{1} / c$ and $C 2 / c$, respectively. The asymmetric unit of 1 contains a single molecule of the complex together with one molecule of co-crystallised ethanol. Compounds 2 and 4 contain four and three independent molecules, respectively in their asymmetric units. The differences in geometric parameters (bond distances and angles) of the independent molecules are however insignificant. In all three complexes the gold atom is bound to the carbon-atom of the IPr-ligand and a selenium or sulfur atom from the deprotonated acylchalcogenourea ligands (Fig. 1-3).

The metal centre is two-coordinate linear with $\mathrm{C}-\mathrm{Au}-\mathrm{Se} / \mathrm{S}$ angles ranging from $174^{\circ}$ to $177^{\circ}$, as expected for gold(I) compounds. The angles at the chalcogen atoms are around $100^{\circ}$, similar to what is observed in the solid-state structures of gold(I) compounds with anionic Se/S-ligands. Furthermore, the gold-carbon bond distances of approximately $2.0 \AA$ are typical values for gold(I) carbenes. There is only one other example of an NHC-Au(I) complex containing an anionic selenium ligand $\left({ }^{-} \mathrm{SeCN}\right)$ which has been structurally characterised. ${ }^{26}$ In this compound the $\mathrm{Au}-\mathrm{Se}$ bond length of $2.414 \AA$ is slightly longer than those found in complexes $\mathbf{1}$ and 2 (around $2.39 \AA$ ).

Thus, in these compounds the typically bidentate chelating (via Se/S and O-atoms) acylchalcogenoureato ligands behave as simple monodentate $\mathrm{Se}^{-}$or $\mathrm{S}^{-}$ligands. The $\operatorname{ArC}(\mathrm{O})$ units are rotated by almost $90^{\circ}$ away from the CSe- and CS-groups which are bound to the gold centre. There is therefore no interaction whatsoever between the oxygen atom of the acylchalcogenoureato ligand and the metal. We may invoke the Pearson hard/soft principle to account for this observation: the soft nature of the

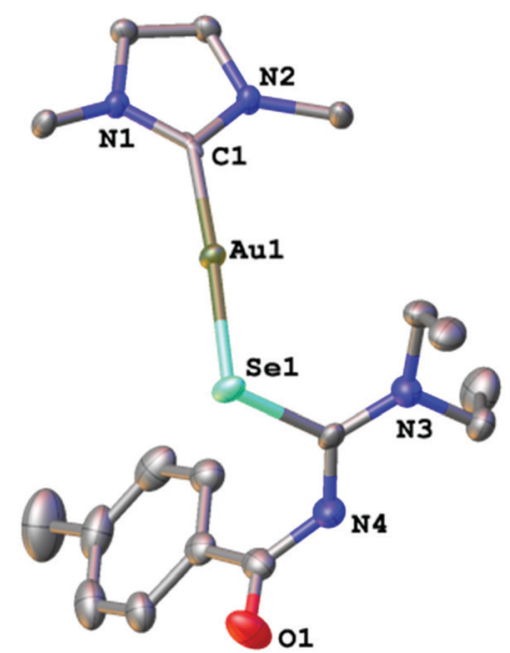

Fig. 1 Molecular structure of 1 . Ellipsoids show 30\% probability levels. Hydrogen atoms as well as the co-crystallised ethanol of solvation have been omitted for clarity. Only the ipso carbon-atoms of the $2,6-{ }^{i} \mathrm{Pr}_{2} \mathrm{C}_{6} \mathrm{H}_{3}$ groups are shown.

gold(I) centre combined with the soft Se- and S-ligand results in a favourable interaction, whereas the hard-soft mismatch between gold and oxygen disfavours any contact between those atoms. This $\kappa \mathrm{Se}, \mathrm{S}$-coordination mode of acylchalcogenoureato ligands is very rare, apart from our previously reported examples containing gold or rhenium, ${ }^{27,40}$ there are no other examples of structurally characterised metal complexes containing Se-bound monoanionic acylselenourea ligands. In the case of the sulfur counterparts, there are three reports in the literature dealing with $\mathrm{kS}$-coordinated acylthioureato ligands (Fig. 4).

An early publication from Richter describes the mercury(II) complexes $\left[\mathrm{Hg}\left\{\mathrm{PhC}(\mathrm{O}) \mathrm{NC}(\mathrm{S}) \mathrm{NR}_{2}\right\}_{2}\right]\left(\mathrm{NR}_{2}=\right.$ morpholine, pyrrolidine) featuring $\mathrm{KS}$-bound ligands (Fig. 4 left) ${ }^{28}$ Although the oxygen atoms are directed towards the metal, the $\mathrm{Hg}-\mathrm{O}$ distance of $2.52 \AA$ was deemed too large for the S,O-chelating mode. The bis(acylthiourea) derived from isophthaloyl dichloride $1,3-\left\{\mathrm{Et}_{2} \mathrm{NC}(\mathrm{S})\right.$ $\mathrm{NHC}(\mathrm{O})\}_{2} \mathrm{C}_{6} \mathrm{H}_{4}$ reacts with two equivalents of $\left[\mathrm{AuCl}\left(\mathrm{PPh}_{3}\right)\right]$ in the presence of base to afford the corresponding bimetallic gold(I) complex (Fig. 4 centre). ${ }^{29}$ More recently, Koch reported the platinum(II) complexes $\left[\mathrm{Pt}(\mathrm{phen})\left\{\operatorname{ArC}(\mathrm{O}) \mathrm{NC}(\mathrm{S}) \mathrm{NR}_{2}\right\}_{2}\right]$ 


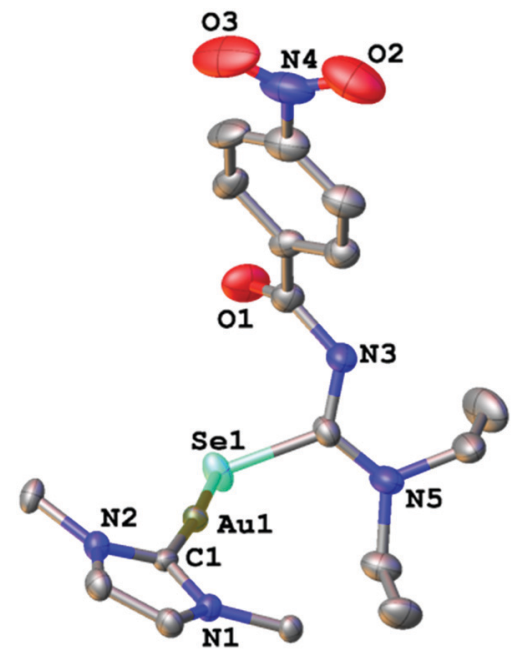

Fig. 2 Molecular structure of one of the independent molecules of 2. Ellipsoids show $30 \%$ probability levels. Hydrogen atoms have been omitted for clarity. Only the ipso carbon-atoms of the 2,6- ${ }^{\mathrm{i}} \mathrm{Pr}_{2} \mathrm{C}_{6} \mathrm{H}_{3}$ groups are shown.

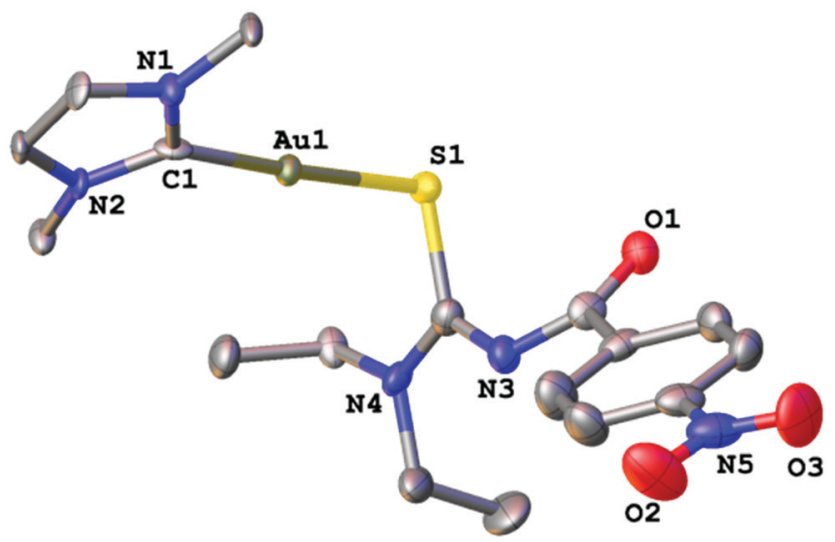

Fig. 3 Molecular structure of one of the independent molecules of 4 Ellipsoids show $30 \%$ probability levels. Hydrogen atoms have been omitted for clarity. Only the ipso carbon-atoms of the $2,6-{ }^{i} \mathrm{Pr}_{2} \mathrm{C}_{6} \mathrm{H}_{3}$ groups are shown.
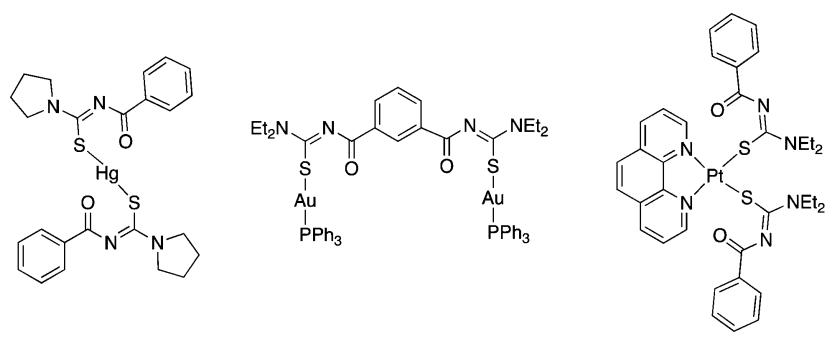

Fig. 4 Known complexes featuring $\kappa S$-coordination of acylthiourea ligands.

(Ar = 1-nap, $\mathrm{R}={ }^{n} \mathrm{Bu} ; \mathrm{Ar}=\mathrm{Ph}, \mathrm{R}=\mathrm{Et}$ ), in which two deprotonated acylthiourea ligands are $\kappa S$-bound to the metal centre (Fig. 4 right). ${ }^{30}$

In summary we disclose here the first examples of gold(I) carbene complexes containing Se- or S-bound acylchalcogenoureato ligands.
The compounds are readily accessible through a one-pot method starting from readily available materials. We are currently exploring the scope of this method with respect to the carbene co-ligand and potential biological activity of this new class of substances.

\section{Experimental}

\section{General}

Reactions were carried out under aerobic conditions using HPLC grade solvents. The acylchalcogenoureas, ${ }^{31-33}[\mathrm{AuCl}(\mathrm{tht})]^{34}$ and the imidazolium salt IPrHCl ${ }^{35}$ were prepared by reported procedures. All other reagents and solvents were procured from commercial suppliers and were used as received. NMR spectra were recorded on Bruker Avance 400 or Bruker Avance III 600 instruments. Spectra were referenced externally to $\mathrm{Me}_{4} \mathrm{Si}\left({ }^{1} \mathrm{H},{ }^{13} \mathrm{C}\right)$ and $\mathrm{Me}_{2} \mathrm{Se}$ $\left({ }^{77} \mathrm{Se}\right)$. Elemental analyses were performed by staff of the in-house elemental analysis facility using an Elementar Vario EL system.

\section{Preparation of the complexes}

To a solution containing the respective acylchalcogenourea, [AuCl(tht)] (1 equiv.) and IPrHCl (1 equiv.) in $\mathrm{MeOH}(15 \mathrm{~mL})$ was added $\mathrm{KO}^{t} \mathrm{Bu}$ (2.2 equiv.). After $c a .2 \mathrm{~h}$ at room temperature, the mixture was evaporated to dryness and the residue was extracted into $\mathrm{CH}_{2} \mathrm{Cl}_{2}(3 \times 10 \mathrm{~mL})$. The combined extracts were filtered through Celite and the filtrate was subsequently dried in vacuum.

[IPrAu$\left.\left\{4-\mathbf{M e C}_{6} \mathbf{H}_{4} \mathbf{C}(\mathbf{O}) \mathbf{N C}(\mathbf{S e}) \mathbf{N E t}_{2}\right\}\right]$ (1). This was prepared as described above from $4-\mathrm{MeC}_{6} \mathrm{H}_{4} \mathrm{C}(\mathrm{O}) \mathrm{NHC}(\mathrm{Se}) \mathrm{NEt}_{2}(0.038 \mathrm{~g}$, $0.128 \mathrm{mmol}$ ), [AuCl(tht)] (0.041 g, $0.128 \mathrm{mmol})$, IPrHCl (0.054 g, $0.128 \mathrm{mmol}$ ) and $\mathrm{KO}^{t} \mathrm{Bu}(0.032 \mathrm{~g}, 0.282 \mathrm{mmol})$. A colourless product was isolated in $75 \%$ yield $(0.085 \mathrm{~g}) .{ }^{1} \mathrm{H}-\mathrm{NMR}(600 \mathrm{MHz}$, $\left.\mathrm{CDCl}_{3}\right): \delta[\mathrm{ppm}]=0.96\left(\mathrm{t}, J=6.9 \mathrm{~Hz}, 6 \mathrm{H}, \mathrm{NCH}_{2} \mathrm{CH}_{3}\right), 1.21(\mathrm{~d}, J=$ $6.9 \mathrm{~Hz}, 12 \mathrm{H}, \mathrm{Me}^{\mathrm{i}} \mathrm{Pr}$ ), 1.25 (d, $J=6.9 \mathrm{~Hz}, 12 \mathrm{H}, \mathrm{Me}-{ }^{\mathrm{i}} \mathrm{Pr}$ ), 2.37 (s, $3 \mathrm{H}, \mathrm{Me}$ ), 2.54 (hept., $J=6.8 \mathrm{~Hz}, 4 \mathrm{H}, \mathrm{CH}^{-}{ }^{\mathrm{i}} \mathrm{Pr}$ ), 3.40 (q, $J=$ $7.1 \mathrm{~Hz}, 4 \mathrm{H}, \mathrm{NCH}_{2} \mathrm{CH}_{3}$ ), 7.09 (d, $\left.J=7.9 \mathrm{~Hz}, 2 \mathrm{H}, \mathrm{C}_{6} \mathrm{H}_{4}\right), 7.15$ (s, $2 \mathrm{H}$, imidazole H), 7.26 (d, $J=7.8 \mathrm{~Hz}, 4 \mathrm{H}, \mathrm{IPr}), 7.50(\mathrm{t}, J=$ $7.8 \mathrm{~Hz}, 2 \mathrm{H}, \mathrm{IPr}), 7.81$ (d, $\left.J=8.1 \mathrm{~Hz}, 2 \mathrm{H}, \mathrm{C}_{6} \mathrm{H}_{4}\right) .{ }^{13} \mathrm{C}-\mathrm{NMR}$ $\left(150 \mathrm{MHz}, \mathrm{CDCl}_{3}\right): \delta[\mathrm{ppm}]=13.5\left(\mathrm{NCH}_{2} \mathrm{CH}_{3}\right), 21.5(\mathrm{Me}), 24.0$ (Me- $\left.{ }^{\mathrm{i}} \mathrm{Pr}\right), 24.4$ (Me- $\left.{ }^{\mathrm{i}} \mathrm{Pr}\right), 28.7\left(\mathrm{CH}^{\mathrm{i}} \mathrm{Pr}\right), 45.1\left(\mathrm{NCH}_{2} \mathrm{CH}_{3}\right), 122.9$ (imidazole C), 124.1 (IPr), $128.1\left(\mathrm{C}_{6} \mathrm{H}_{4}\right), 129.8$ (IPr), 130.5 (IPr), 134.1 (IPr), $135.2\left(\mathrm{C}_{6} \mathrm{H}_{4}\right), 140.3\left(\mathrm{C}_{6} \mathrm{H}_{4}\right), 145.7$ (IPr), 153.2 (CSe), $175.3(\mathrm{CO}), 186.8(\mathrm{CAu}) .{ }^{77} \mathrm{Se}-\mathrm{NMR}\left(76 \mathrm{MHz}^{\mathrm{CDCl}}{ }_{3}\right): \delta[\mathrm{ppm}]=$ 105.1. Elemental analyses calcd for $\mathrm{C}_{40} \mathrm{H}_{53} \mathrm{~N}_{4} \mathrm{OSeAu}$ (881.8): C 54.48, H 6.06, N 6.35; found: C 54.13, H 6.09, N 6.25\%. X-ray quality crystals were obtained by vapour diffusion of hexanes into an ethanolic solution of the compound.

[IPrAu $\left\{4-\mathbf{O}_{2} \mathbf{N C}_{6} \mathbf{H}_{4} \mathbf{C}(\mathbf{O}) \mathbf{N C}\left(\mathrm{Se}_{\mathbf{N}} \mathbf{N E t}_{2}\right\}\right]$ (2). This was prepared as described above from $4-\mathrm{O}_{2} \mathrm{NC}_{6} \mathrm{H}_{4} \mathrm{C}(\mathrm{O}) \mathrm{NHC}(\mathrm{Se}) \mathrm{NEt}_{2}(0.041 \mathrm{~g}$, $0.125 \mathrm{mmol}),[\mathrm{AuCl}(\mathrm{tht})](0.040 \mathrm{~g}, 0.125 \mathrm{mmol}), \operatorname{IPrHCl}(0.053 \mathrm{~g}$, $0.125 \mathrm{mmol})$ and $\mathrm{KO}^{t} \mathrm{Bu}(0.031 \mathrm{~g}, 0.275 \mathrm{mmol})$. A yellow product was isolated in $73 \%$ yield (0.083 g). ${ }^{1} \mathrm{H}-\mathrm{NMR}\left(400 \mathrm{MHz}, \mathrm{CDCl}_{3}\right)$ : $\delta[\mathrm{ppm}]=0.98\left(\mathrm{~m}, 6 \mathrm{H}, \mathrm{NCH}_{2} \mathrm{CH}_{3}\right), 1.20$ (m, $\left.24 \mathrm{H}, \mathrm{Me}-{ }^{\mathrm{i}} \mathrm{Pr}\right), 2.51$ (m, $4 \mathrm{H}, \mathrm{CH}^{-}{ }^{\mathrm{i}} \mathrm{Pr}$ ), 3.46 (q, $\left.J=7.1 \mathrm{~Hz}, 4 \mathrm{H}, \mathrm{IPr}\right), 7.16(\mathrm{~s}, 2 \mathrm{H}$, imidazole H), 7.24 (d, $J=7.8 \mathrm{~Hz}, 4 \mathrm{H}, \mathrm{IPr}), 7.48$ (t, $J=7.8 \mathrm{~Hz}, 2 \mathrm{H}$, IPr), $8.04\left(\mathrm{~d}, J=8.9 \mathrm{~Hz}, 2 \mathrm{H}, \mathrm{C}_{6} \mathrm{H}_{4}\right), 8.12\left(\mathrm{~d}, J=8.9 \mathrm{~Hz}, 2 \mathrm{H}, \mathrm{C}_{6} \mathrm{H}_{4}\right)$. 
${ }^{13} \mathrm{C}-\mathrm{NMR}\left(100 \mathrm{MHz}, \mathrm{CDCl}_{3}\right): \delta[\mathrm{ppm}]=13.3\left(\mathrm{NCH}_{2} \mathrm{CH}_{3}\right), 23.9$ (Me- $\left.{ }^{\mathrm{i}} \mathrm{Pr}\right), 24.3$ (Me- $\left.{ }^{\mathrm{i}} \mathrm{Pr}\right), 28.7\left(\mathrm{CH}^{\mathrm{i}} \mathrm{Pr}\right), 45.4\left(\mathrm{NCH}_{2} \mathrm{CH}_{3}\right), 122.6$ $\left(\mathrm{C}_{6} \mathrm{H}_{4}\right), 123.0$ (imidazole C), 124.1 (IPr), $130.2\left(\mathrm{C}_{6} \mathrm{H}_{4}\right), 130.5$ (IPr), 134.0 (IPr), $144.4\left(\mathrm{C}_{6} \mathrm{H}_{4}\right), 145.6$ (IPr), $148.8\left(\mathrm{C}_{6} \mathrm{H}_{4}\right), 158.8$ (CSe), 171.2 (CO), 186.1 (CAu). ${ }^{77} \mathrm{Se}-\mathrm{NMR}\left(76 \mathrm{MHz}, \mathrm{CDCl}_{3}\right): \delta[\mathrm{ppm}]=$ 122.4. Elemental analyses calcd for $\mathrm{C}_{39} \mathrm{H}_{50} \mathrm{~N}_{5} \mathrm{O}_{3} \mathrm{SeAu}$ (912.8): C 51.32, H 5.52, N 7.67; found: C 51.28, H 5.44, N 7.52\%. X-ray quality crystals were obtained by vapour diffusion of hexanes into a methanolic solution of the compound.

[IPrAu\{4-MeC $\left.\left.\mathbf{H}_{6} \mathbf{H}_{4} \mathbf{C}(\mathbf{O}) \mathbf{N C}(\mathbf{S}) \mathbf{N E t}_{2}\right\}\right]$ (3). This was prepared as described above from $4-\mathrm{MeC}_{6} \mathrm{H}_{4} \mathrm{C}(\mathrm{O}) \mathrm{NHC}(\mathrm{S}) \mathrm{NEt}_{2}(0.034 \mathrm{~g}$, $0.136 \mathrm{mmol}$ ), [AuCl(tht)] (0.044 g, $0.136 \mathrm{mmol}), \operatorname{IPrHCl}(0.058 \mathrm{~g}$, $0.136 \mathrm{mmol})$ and $\mathrm{KO}^{t} \mathrm{Bu}(0.034 \mathrm{~g}, 0.299 \mathrm{mmol})$. A colourless product was isolated in $61 \%$ yield $(0.070 \mathrm{~g}) .{ }^{1} \mathrm{H}-\mathrm{NMR}(400 \mathrm{MHz}$, $\left.\mathrm{CDCl}_{3}\right): \delta[\mathrm{ppm}]=0.96\left(\mathrm{t}, J=7.1 \mathrm{~Hz}, 6 \mathrm{H}, \mathrm{NCH}_{2} \mathrm{CH}_{3}\right), 1.19(\mathrm{~d}, J=$ $6.9 \mathrm{~Hz}, 12 \mathrm{H}, \mathrm{Me}^{-}{ }^{\mathrm{i}} \mathrm{Pr}$ ), 1.21 (d, $J=6.9 \mathrm{~Hz}, 12 \mathrm{H}, \mathrm{Me}^{\mathrm{i}} \mathrm{Pr}$ ), 2.37 (s, $3 \mathrm{H}, \mathrm{Me}$ ), 2.50 (hept., $J=6.9 \mathrm{~Hz}, 4 \mathrm{H}, \mathrm{CH}^{-}{ }^{\mathrm{i}} \mathrm{Pr}$ ), 3.41 (q, $J=$ $7.1 \mathrm{~Hz}, 4 \mathrm{H}, \mathrm{IPr}), 7.07$ (d, $\left.J=7.9 \mathrm{~Hz}, 2 \mathrm{H}, \mathrm{C}_{6} \mathrm{H}_{4}\right), 7.14(\mathrm{~s}, 2 \mathrm{H}$, imidazole $\mathrm{H}$ ), 7.24 (d, $J=7.8 \mathrm{~Hz}, 4 \mathrm{H}, \mathrm{IPr}), 7.48(\mathrm{t}, J=7.8 \mathrm{~Hz}$, $2 \mathrm{H}, \mathrm{IPr}), 7.79$ (d, $\left.J=8.1 \mathrm{~Hz}, 2 \mathrm{H}, \mathrm{C}_{6} \mathrm{H}_{4}\right) .{ }^{13} \mathrm{C}-\mathrm{NMR}(150 \mathrm{MHz}$, $\left.\mathrm{CDCl}_{3}\right): \delta[\mathrm{ppm}]=13.4\left(\mathrm{NCH}_{2} \mathrm{CH}_{3}\right), 21.5(\mathrm{Me}), 23.9\left(\mathrm{Me}-{ }^{\mathrm{i}} \mathrm{Pr}\right)$, 24.4 (Me- $\left.{ }^{\mathrm{i}} \mathrm{Pr}\right), 28.7\left(\mathrm{CH}-{ }^{\mathrm{i}} \mathrm{Pr}\right), 44.4\left(\mathrm{NCH}_{2} \mathrm{CH}_{3}\right), 123.0$ (imidazole C), 124.1 (IPr), $128.0\left(\mathrm{C}_{6} \mathrm{H}_{4}\right), 129.6$ (IPr), 130.5 (IPr), 134.0 (IPr), $135.7\left(\mathrm{C}_{6} \mathrm{H}_{4}\right), 139.9\left(\mathrm{C}_{6} \mathrm{H}_{4}\right), 145.6$ (IPr), 163.7 (CS), 174.1 (CO), 184.2 (CAu). Elemental analyses calcd for $\mathrm{C}_{40} \mathrm{H}_{53} \mathrm{~N}_{4} \mathrm{OSAu}$ (834.9): $\mathrm{C}$ 57.54, $\mathrm{H}$ 6.71, $\mathrm{N}$ 6.84; found: $\mathrm{C}$ 56.85, $\mathrm{H}$ 6.32, N 6.70\%.

[IPrAu $\left.\left\{4-\mathrm{O}_{2} \mathbf{N C}_{6} \mathbf{H}_{4} \mathbf{C}(\mathbf{O}) \mathbf{N C}(\mathbf{S}) \mathbf{N E t}_{2}\right\}\right]$ (4). This was prepared as described above from $4-\mathrm{O}_{2} \mathrm{NC}_{6} \mathrm{H}_{4} \mathrm{C}(\mathrm{O}) \mathrm{NHC}(\mathrm{S}) \mathrm{NEt}_{2}(0.030 \mathrm{~g}$, $0.107 \mathrm{mmol}),[\mathrm{AuCl}(\mathrm{tht})](0.034 \mathrm{~g}, 0.107 \mathrm{mmol}), \operatorname{IPrHCl}(0.046 \mathrm{~g}$, $0.107 \mathrm{mmol})$ and $\mathrm{KO}^{t} \mathrm{Bu}(0.026 \mathrm{~g}, 0.235 \mathrm{mmol})$. A yellow product was isolated in $85 \%$ yield $(0.079 \mathrm{~g}) .{ }^{1} \mathrm{H}-\mathrm{NMR}$ (600 $\left.\mathrm{MHz}, \mathrm{CDCl}_{3}\right)$ : $\delta[\mathrm{ppm}]=1.01\left(\mathrm{t}, J=6.9 \mathrm{~Hz}, 6 \mathrm{H}, \mathrm{NCH}_{2} \mathrm{CH}_{3}\right), 1.16-1.20(\mathrm{~m}, 24 \mathrm{H}$, Me- ${ }^{\mathrm{i}} \mathrm{Pr}$ ), 2.48 (hept., $J=6.9 \mathrm{~Hz}, 4 \mathrm{H}, \mathrm{CH}^{\mathrm{i}} \mathrm{Pr}$ ), 3.48 (q, $J=7.0 \mathrm{~Hz}$, $4 \mathrm{H}, \mathrm{IPr}$ ), 7.15 (s, $2 \mathrm{H}$, imidazole H), 7.23 (d, $J=7.8 \mathrm{~Hz}, 4 \mathrm{H}, \mathrm{IPr}$ ), 7.46 (t, $J=7.8 \mathrm{~Hz}, 2 \mathrm{H}, \mathrm{IPr}), 8.03\left(\mathrm{~d}, J=8.9 \mathrm{~Hz}, 2 \mathrm{H}, \mathrm{C}_{6} \mathrm{H}_{4}\right), 8.11$ $\left(\mathrm{d}, J=8.9 \mathrm{~Hz}, 2 \mathrm{H}, \mathrm{C}_{6} \mathrm{H}_{4}\right) .{ }^{13} \mathrm{C}-\mathrm{NMR}\left(150 \mathrm{MHz}, \mathrm{CDCl}_{3}\right): \delta[\mathrm{ppm}]=$ $13.1\left(\mathrm{NCH}_{2} \mathrm{CH}_{3}\right), 23.8$ (Me- $\left.{ }^{\mathrm{i}} \mathrm{Pr}\right), 24.3\left(\mathrm{Me}-{ }^{\mathrm{i}} \mathrm{Pr}\right), 28.6$ (CH- $\left.{ }^{\mathrm{i}} \mathrm{Pr}\right), 45.1$ $\left(\mathrm{NCH}_{2} \mathrm{CH}_{3}\right.$ ), 122.5 (imidazole C), 123.1 (IPr), 124.1 (IPr), 130.1 (IPr), $130.6\left(\mathrm{C}_{6} \mathrm{H}_{4}\right), 133.9$ (IPr), 145.0 (IPr), $145.6\left(\mathrm{C}_{6} \mathrm{H}_{4}\right), 148.5$ $\left(\mathrm{C}_{6} \mathrm{H}_{4}\right), 169.3(\mathrm{CS}), 170.9(\mathrm{CO}), 183.4(\mathrm{CAu})$. Elemental analyses calcd for $\mathrm{C}_{39} \mathrm{H}_{50} \mathrm{~N}_{5} \mathrm{O}_{3} \mathrm{SAu}$ (865.9): C 54.10, H 5.82, N 8.09; found: C 53.95, H 5.68, N 7.88\%. X-ray quality crystals were obtained by vapour diffusion of hexanes into a methanolic solution of the compound.

\section{X-ray crystallography}

Diffraction data for complexes $\mathbf{2}$ and $\mathbf{4}$ were collected using an Enraf-Nonius KappaCCD system located in front of a FR591 rotating anode equipped with an Incoatec focusing Montel optic for Mo radiation and an Oxford Cryosystems Cryostream 700. For data integration the EVAL-14 package ${ }^{36}$ was employed. For data scaling and absorption correction (combination of Gaussian and multiscan corrections) SADABS was used. Diffraction data for complex 1 was collected using a Rigaku Oxford Diffraction Gemini E Ultra diffractometer, equipped with an EOS CCD area detector and a four-circle kappa goniometer. Data integration, scaling and empirical absorption correction was carried out using the CrysAlis Pro program package. ${ }^{37}$ All crystal structures were solved using SHELXT $^{38}$ and refined using SHELXL. ${ }^{38}$ The Olex2 graphical user interface ${ }^{39}$ was used for all structure manipulations and for molecular graphics. Crystallographic and refinement details are collected in Table 1.

Table 1 Crystallographic and refinement details for complexes 1, 2 and 4

\begin{tabular}{|c|c|c|c|}
\hline & $1 \mathrm{EtOH}$ & 2 & 4 \\
\hline CCDC code & 1907692 & 1907635 & 1907634 \\
\hline Empirical formula & $\mathrm{C}_{42} \mathrm{H}_{59} \mathrm{AuN}_{4} \mathrm{O}_{2} \mathrm{Se}$ & $\mathrm{C}_{39} \mathrm{H}_{50} \mathrm{AuN}_{5} \mathrm{O}_{3} \mathrm{Se}$ & $\mathrm{C}_{39} \mathrm{H}_{50} \mathrm{AuN}_{5} \mathrm{O}_{3} \mathrm{~S}$ \\
\hline Colour & Colourless & Yellow & Colourless \\
\hline Formula weight $\left(\mathrm{g} \mathrm{mol}^{-1}\right)$ & 927.85 & 912.76 & 865.86 \\
\hline Temperature $(\mathrm{K})$ & $150(2)$ & $100(2)$ & $100(2)$ \\
\hline Wavelength $(\AA)$ & 0.71073 & 0.71073 & 0.71073 \\
\hline Crystal system & Monoclinic & Monoclinic & Monoclinic \\
\hline Space group & $P 2_{1} / n$ & $P 2_{1} / c$ & $C 2 / c$ \\
\hline \multirow[t]{4}{*}{ Unit cell dimensions } & $a=14.1899(3) \AA$ & $a=19.003(7) \AA$ & $a=33.860(7) \AA$ \\
\hline & $b=16.2803(4) \AA$ & $b=21.806(3) \AA$ & $b=36.211(7) \AA$ \\
\hline & $c=18.4131(5) \AA$ & $c=37.911(6) \AA$ & $c=19.899(3) \AA$ \\
\hline & $\beta=101.693(2)^{\circ}$ & $\beta=91.39(2)^{\circ}$ & $\beta=107.145(18)^{\circ}$ \\
\hline Volume & $4165.47(18) \AA^{3}$ & $15705(7) \AA^{3}$ & $23314(8) \AA^{3}$ \\
\hline$Z$ & 4 & 16 & 24 \\
\hline$\rho_{\text {calc }}$ & $1.480 \mathrm{~g} \mathrm{~cm}^{-3}$ & $1.544 \mathrm{~g} \mathrm{~cm}^{-3}$ & $1.480 \mathrm{~g} \mathrm{~cm}^{-3}$ \\
\hline$\mu$ & $4.444 \mathrm{~mm}^{-1}$ & $4.716 \mathrm{~mm}^{-1}$ & $3.882 \mathrm{~mm}^{-1}$ \\
\hline$F(000)$ & 1872.0 & 7296.0 & 10512 \\
\hline Crystal size & $0.03 \times 0.06 \times 0.12 \mathrm{~mm}$ & $0.05 \times 0.12 \times 0.18 \mathrm{~mm}$ & $0.04 \times 0.05 \times 0.10 \mathrm{~mm}$ \\
\hline$\theta$ range for data collection & 4.758 to $59.144^{\circ}$ & 2.599 to $32.974^{\circ}$ & 2.646 to $28.300^{\circ}$ \\
\hline Reflections collected & 25188 & 405585 & 133811 \\
\hline Independent reflections & 9924 & 58942 & 28915 \\
\hline Parameters & 464 & 1805 & 1350 \\
\hline Goodness-of-fit on $F^{2}$ & 1.100 & 1.029 & 1.007 \\
\hline Final $\mathrm{R}$ indices $[I>2 \sigma(\mathrm{I})]$ & $R_{1}=0.0388$ & $R_{1}=0.0614$ & $\mathrm{R}_{1}=0.0659$ \\
\hline & $\mathrm{w} R_{2}=0.0784$ & $\mathrm{w} R_{2}=0.1157$ & $\mathrm{w} R_{2}=0.1048$ \\
\hline Largest difference peak/hole & $3.06 /-1.38{\mathrm{e} \AA^{-3}}^{-3}$ & $6.7 /-3.6{\mathrm{e} \AA^{-3}}^{-3}$ & $1.4 /-2.0{\mathrm{e} \AA^{-3}}^{-3}$ \\
\hline
\end{tabular}




\section{Conflicts of interest}

There are no conflicts to declare.

\section{Acknowledgements}

We thank RETORTE GmbH for a generous gift of selenium metal.

\section{References}

1 L. Beyer, R. Kirmse and E. Hoyer, Z. Chem., 1975, 15, 197.

2 R. Kirmse, L. Beyer and E. Hoyer, Z. Chem., 1975, 15, 454-455.

3 R. Kirmse, L. Beyer and E. Hoyer, Chem. Phys. Lett., 1977, 49, 544-546.

4 J. Stach, R. Kirmse, A. Heinrich, W. Dietzsch, J. Hartung and L. Beyer, Z. Chem., 1983, 23, 453-454.

5 M. Kampf, R. Richter, S. Gerber and R. Kirmse, Z. Anorg. Allg. Chem., 2004, 630, 1437-1443.

6 M. Kampf, R. Richter, L. Hennig, A. Eidner, J. Baldamus and R. Kirmse, Z. Anorg. Allg. Chem., 2004, 630, 2677-2686.

7 M. Kampf, R. Richter, J. Griebel, A. Weller and R. Kirmse, Z. Anorg. Allg. Chem., 2005, 631, 698-708.

8 J. C. Bruce and K. R. Koch, Acta Crystallogr., Sect. C: Cryst. Struct. Commun., 2008, 64, m1-m4.

9 W. Bensch and M. Schuster, Z. Anorg. Allg. Chem., 1993, 619, 786-790.

10 W. Bensch and M. Schuster, Z. Anorg. Allg. Chem., 1993, 619, 791-795.

11 M. Akhtar, J. Akhtar, M. A. Malik, F. Tuna, M. Helliwell and P. O’Brien, J. Mater. Chem., 2012, 22, 14970-14975.

12 W. Bensch and M. Schuster, Z. Anorg. Allg. Chem., 1994, 620, 1479-1482.

13 M. Schuster and W. Bensch, Z. Anorg. Allg. Chem., 1994, 620, 737-742.

14 J. Akhtar, M. A. Malik, S. K. Stubbs, P. O’Brien, M. Helliwell and D. J. Binks, Eur. J. Inorg. Chem., 2011, 2984-2990.

15 J. Akhtar, M. Akhtar, M. A. Malik, P. O'Brien and J. Raftery, J. Am. Chem. Soc., 2012, 134, 2485-2487.

16 A. Molter, J. Rust, C. W. Lehmann and F. Mohr, Tetrahedron, 2012, 68, 10586-10591.

17 M. Dörner, J. M. Rautiainen, J. Rust, C. W. Lehmann and F. Mohr, Eur. J. Inorg. Chem., 2017, 789-797.
18 A. Molter, S. Kathrein, B. Kircher and F. Mohr, Dalton Trans., 2018, 47, 5055-5064.

19 J. Kuchar, J. Rust, C. W. Lehmann and F. Mohr, Eur. J. Inorg. Chem., 2018, 5215-5222.

20 B. David, U. Monkowius, J. Rust, C. W. Lehmann, L. Hyzak and F. Mohr, Dalton Trans., 2014, 43, 11059-11066.

21 A. Molter and F. Mohr, Coord. Chem. Rev., 2010, 254, 19-45.

22 S. Gaillard, A. M. Z. Slawin and S. P. Nolan, Chem. Commun., 2010, 46, 2742-2744.

23 P. de Frémont, N. M. Scott, E. D. Stevens and S. P. Nolan, Organometallics, 2005, 24, 2411-2418.

24 E. Schuh, C. Pflüger, A. Citta, A. Folda, M. P. Rigobello, A. Bindoli, A. Casini and F. Mohr, J. Med. Chem., 2012, 55, 5518-5528.

25 R. Rubbiani, E. Schuh, A. Meyer, J. Lemke, J. Wimberg, N. Metzler-Nolte, F. Meyer, F. Mohr and I. Ott, MedChemComm, 2013, 4, 942-948.

26 M. V. Baker, P. J. Barnard, S. K. Brayshaw, J. L. Hickey, B. W. Skelton and A. H. White, Dalton Trans., 2005, 37-43.

27 A. Molter, J. Rust, C. W. Lehmann and F. Mohr, Tetrahedron, 2012, 68, 10586-10591.

28 R. Richter, J. Seiler, L. Beyer, O. Lindqvist and L. Andersen, Z. Anorg. Allg. Chem., 1985, 522, 171-183.

29 V. D. Schwade, L. Kirsten, A. Hagenbach, E. Schulz Lang and U. Abram, Polyhedron, 2013, 55, 155-161.

30 I. A. Kotzé, V. J. Smith, E. F. Kangara and K. R. Koch, New J. Chem., 2017, 41, 14995-15002.

31 I. B. Douglass, J. Am. Chem. Soc., 1937, 59, 740-742.

32 A. Molter, J. Rust, C. W. Lehmann and F. Mohr, ARKIVOC, 2011, vi, 10-17.

33 K. H. König, L. Kaul, M. Kuge and M. Schuster, Liebigs Ann. Chem., 1987, 1115-1116.

34 R. Usón, A. Laguna and M. Laguna, Inorg. Synth., 1989, 26, 85-91.

35 L. Hintermann, Beilstein J. Org. Chem., 2007, 3, 1-5.

36 A. J. M. Duisenberg, L. M. J. Kroon-Batenburg and A. M. M. Schreurs, J. Appl. Crystallogr., 2003, 36, 220-229.

37 CrysAlisPro, Rigaku Oxford Diffraction Ltd, Oxford, UK, 2016.

38 G. M. Sheldrick, Acta Crystallogr., Sect. C: Struct. Chem., 2015, 71, 3-8.

39 O. V. Dolomanov, L. J. Bourhis, R. J. Gildea, J. A. K. Howard and H. Puschmann, J. Appl. Crystallogr., 2009, 42, 339-341.

40 V. D. Schwade, A. Hagenbach, E. S. Lang, K. Klauke, F. Mohr and U. Abram, Eur. J. Inorg. Chem., 2014, 1949-1954. 